\title{
Upregulation of LncDQ is Associated with Poor Prognosis and Promotes Tumor Progression via Epigenetic Regulation of the EMT Pathway in HCC
}

\author{
Bing Zeng ${ }^{\mathrm{a}, \mathrm{c}}$ Zewei Lin ${ }^{\mathrm{b}}$ Huilin Ye $\mathrm{Z}^{\mathrm{c}}$ Di Cheng ${ }^{\mathrm{c}}$ Guangtao Zhang ${ }^{\mathrm{b}}$ Jindu Zhou ${ }^{\mathrm{d}}$ \\ Zhifeng Huang $^{\mathrm{b}}$ Meng Wang ${ }^{\mathrm{b}} \quad$ Canfeng Cai $^{\mathrm{a}} \quad$ Jun Zeng ${ }^{\mathrm{a}} \quad$ Chaoming Tang $^{\mathrm{a}}$ \\ Jikui Liub \\ aDepartment of Gastrointestinal Surgery, The Sixth Affiliated Hospital of Guangzhou Medical \\ University, Qing Yuan People's Hospital, Guangdong, 'Department of Hepatobiliary Surgery, Peking \\ University Shenzhen Hospital, Guangdong, 'Department of Hepatopancreatobiliary Surgery, Sun Yat- \\ sen Memorial Hospital of Sun Yat-sen University, Guangzhou, dDepartment of Hepatobiliary Surgery, \\ Shenzhen Guangming New District People's Hospital, Guangdong, China
}

\section{Key Words}

$\mathrm{LncDQ} \cdot \mathrm{EZH} 2 \cdot$ Epithelial-mesenchymal transition • Hepatocellular carcinoma

\begin{abstract}
Background/Aims: Long noncoding RNAs (IncRNAs) are key regulators of cancer initiation and progression. In this study, we investigated the clinical value and functional role of LncRNA DQ786243 (LnCDQ) in the pathogenesis of hepatocellular carcinoma (HCC). Methods: To investigate the expression level of LnCDQ in HCC, we performed quantitative real-time PCR using total RNA extracted from HCC tumor tissues and their matched nonneoplastic counterparts, as well as from the serum of HCC patients and healthy volunteers. The correlation of LncDQ expression with clinicopathologic features and prognosis was analyzed. The functional role of LnCDQ in cell proliferation, migration, and invasion were evaluated by MTT cell viability, wound healing, and transwell assays in vitro and in vivo. RNA immunoprecipitation and chromatin immunoprecipitation assays were performed to analyze the potential mechanism of LnCDQ in HCC cells. Results: LncDQ was upregulated in both HCC tissue samples and serum and was correlated with low survival rate and adverse clinical pathological characteristics. Multivariate analysis demonstrated that LnCDQ expression was an independent prognostic factor for HCC. The area under the receiver operating characteristic curve was 0.804 with a sensitivity of 0.72 and a specificity of 0.8 . Knockdown of LncDQ induced inhibition of cell proliferation, migration, and invasion in vitro and in vivo. Mechanistically, LncDQ regulated the epithelial-mesenchymal transition pathway by interacting with $\mathrm{EZH}$, to
\end{abstract}

B. Zeng, Z. Lin and H. Ye contributed equally to this work.

Jikui Liu, Jun Zeng

and Chaoming Tang
Department of Hepatobiliary Surgery, Peking University Shenzhen Hospital,

The Sixth Affiliated Hospital of Guangzhou Medical University, Qing Yuan People's Hospital (China)

E-Mail liujk8929@163.com, chaomingtt001@163.com 
epigenetically repress the expression of E-cadherin in HCC cells. Conclusions: Taken together, the results of our study indicate that LnCDQ plays a critical role in HCC progression, and may serve as a potential diagnostic and prognostic biomarker for HCC.

(C) 2018 The Author(s)

Published by S. Karger AG, Basel

\section{Introduction}

Hepatocellular carcinoma (HCC) is an aggressive tumor that accounts for $70-90 \%$ of primary liver cancers [1]. The prognosis of HCC is poor due to the lack of a typical clinical presentation in the early stage. HCC has a high rate of intrahepatic metastasis and there is typically rapid recurrence after resection [2]. To date, few reliable markers are available to accurately diagnosis and predict HCC prognosis. Furthermore, some existing molecular markers, such as alpha-fetoprotein (AFP), have not yielded satisfactory results, giving false negatives in 30-40\% of patients with HCC [3]. Therefore, biomarkers that can help diagnosis or predict tumor progression and prognosis are needed, as they would serve as an important decision-making tool.

Long noncoding RNAs (lncRNAs) are a class of long transcripts that lack protein-coding potential [4], and play vital roles in diverse diseases [5-8]. Importantly, they are involved in the biological processes of cancer cells, including cell proliferation, invasion, and metastasis [9]. It is widely documented that the epithelial-mesenchymal transition (EMT) plays key roles in tumor progression [10]. Interestingly, a number of studies have demonstrated that lncRNAs are involved in regulation of the EMT.

Aberrant expression of IncRNAs has been observed in many types of cancers [1113]. Previous profile studies of IncRNA expression have documented the dysregulation of a series of lncRNAs in HCC including LncRNA DQ786243 (LncDQ) [14]. A recent study demonstrated that LncDQ is upregulated in active Crohn's disease (CD) and correlates with disease severity [15]. Sun et al. [16] found that LncDQ is increased in colorectal cancer, and LncDQ overexpression promotes the cell proliferation and metastasis of colorectal cancer cells both in vitro and in vivo. However, the clinical value and role of LncDQ in HCC have not been investigated.

In this study, we investigated whether LncDQ was upregulated in HCC and investigated its clinical value as a biomarker of HCC. Furthermore, the effects of LncDQ expression on HCC cell behavior were evaluated both in vitro and in vivo. Mechanistic analysis revealed that LncDQ repressed E-cadherin expression by binding to the histone-lysine $N$-methyltransferase, enhancer of zeste homology 2 (EZH2), leading to EMT promotion in HCC cells. Our study indicated that LncDQ may be useful as a novel diagnostic and prognostic biomarker for HCC, providing a potential therapeutic target for controlling HCC progression.

\section{Materials and Methods}

\section{Clinical samples and cell lines}

Clinical HCC tumor tissues, corresponding adjacent non-tumor tissues ( $\mathrm{n}=84$ ), and serum samples $(\mathrm{n}=50)$ were obtained from patients who underwent radical resection at Peking University Shenzhen Hospital and Sun Yat-sen Memorial Hospital between 2008 and 2012. The diagnosis of HCC was confirmed by histopathology. The control serum samples $(n=30)$ were randomly selected from patients undergoing routine health check-ups who did not have any disease. Written consent approving the use of their samples for research purposes was obtained from each patient. The study was approved by the Institute Research Ethics Committee at Peking University Shenzhen Hospital and Sun Yat-sen Memorial Hospital. The HCC cell lines (Huh-7, HepG2, HepG3B, and SMMC7721) and normal cell line (L02) were maintained in Dulbecco's Modified Eagle Medium (DMEM) supplemented with 10\% fetal bovine serum (FBS; Gibco, Grand Island, $\mathrm{NY}$ ). All cell lines were maintained at $37^{\circ} \mathrm{C}$ in a humidified atmosphere with $5 \% \mathrm{CO}_{2}$. 


\section{Cellular Physiology Cell Physiol Biochem 2018;46:1122-1133 \begin{tabular}{l|l} 
DOI: 10.1159/000488841 & $\begin{array}{l}\text { O } 2018 \text { The Author(s). Published by S. Karger AG, Basel } \\
\text { wwww.karger.com/cpb }\end{array}$
\end{tabular} \\ Zeng et al.: LncDQ Promotes HCC Progression via EMT Pathway}

\section{RNA extraction and quantitative real-time PCR}

Tissue samples and serum RNA were extracted with TRIzol reagent and TRIzol LS reagent (Invitrogen, Carlsbad, CA) respectively, according to the manufacturer's protocol. Total RNA was reversed using the PrimeScript RT Reagent Kit with gDNA Eraser (Takara, Dalian, China). Quantitative real-time PCR (qRT-PCR) was performed in the LightCycler480 System using the SYBR Premix ExTaq Kit (Takara). The primers used were as follows: LncDQ forward, 5'-TAG GCG GAC ATT GTG GTG AGT-3' and reverse, 5'-CTT CTG CTG GGC TGT TGA GTG-3'. E-cadherin forward, 5'-TAG AGG GTC ACC GCG TCT AT-3' and reverse, 5'-CGT ACC GCT GAT TGG CTG AG-3'. GAPDH forward, 5'-AGC CAC ATC GCT CAG ACA C-3' and reverse, 5'-GCC CAA TAC GAC CAA ATC C- $3^{\prime}$. The relative expression levels were calculated by the $2^{-\Delta \Delta} \mathrm{CT}$ method. Each assay was performed in triplicate.

\section{Cell transfection}

Three small interfering RNAs (siRNAs) and negative control siRNA (si-NC) were purchased from GenePharma Co. (Shanghai, China). The target sequences for LncDQ were as follows: si-LncDQ-1, 5'-GCC ATG GGT ACC CGG ATG ATG TTAT-3', si-LncDQ-2 5'-CCA TGG GTA CCC GGA TGA TGT TATA-3'. HepG2 and SMMC7721 cells were grown on 6-well plates to confluency and transfected with LncDQ siRNA or si-NC using Lipofectamine ${ }^{\mathrm{TM}}$ RNAiMAX according to the manufacturer's instructions. After $24 \mathrm{~h}$ of transfection, cells were collected for qRT-PCR and western blot analyses.

\section{Western blot analysis}

Cell cytosolic protein fractions were extracted using RIPA buffer (Beyotime Biotechnology, Shanghai, China) according to the manufacturer's protocol. Briefly, proteins were separated by sodium dodecyl sulfate polyacrylamide gel electrophoresis and then transferred to polyvinylidene fluoride membranes. After blocking in 5\% nonfat milk, the membranes were incubated with the following primary antibodies: E-cadherin, N-cadherin, vimentin, and EZH2 antibodies (Abcam, Cambridge, UK), according to the manufacturer's instructions. $\beta$-actin antibody (Boster Biological Technology, Pleasanton, CA) was used as the control.

\section{Cell proliferation assays}

Cell proliferation was performed with the MTT cell viability assay. Cells were seeded in 96-well plates at a density of 1,000 cells per well. At $24,48,72$, and 96 h, the cells were stained with $20 \mu \mathrm{L}$ MTT $(0.5 \mathrm{mg} /$ $\mathrm{mL}$ ), and $100 \mu \mathrm{L}$ dimethyl sulfoxide was added. The absorbance was measured at $490 \mathrm{~nm}$. The anchorageindependent sphere formation assay was performed by culturing the cells in suspension in serum-free DMEM-F12 supplemented with B27 (Invitrogen), epidermal growth factor (BD Biosciences, San Jose, CA), bovine serum albumin (Sigma, St. Louis, MO), and insulin (Sigma).

\section{Wound healing assay}

Cells were plated in 6-well plates the day before transfection. After $24 \mathrm{~h}$, the transfected cells were wounded with a pipette tip, washed with phosphate-buffered saline, and then allowed to migrate for $24 \mathrm{~h}$. Cell migration images were photographed using an inverted microscope. The results were expressed using a migration index as previously described [17].

\section{Matrigel invasion assay}

Cells were collected and suspended in $100 \mu \mathrm{L}$ serum-free DMEM, and then transferred into the upper chamber coated with matrigel. In the lower chamber, 10\% FBS-containing medium served as the chemoattractant. After several hours of incubation, the cells that did not invade through the pores were removed. Then the inserts were stained with $0.2 \%$ crystal violet, imaged, and counted under an inverted microscope.

\section{RNA immunoprecipitation}

RNA immunoprecipitation (RIP) experiments were performed using the Magna RIP RNA-Binding Protein Immunoprecipitation Kit (Millipore, Stafford, VA) according to the manufacturer's instructions. Antibodies for RIP assays against EZH2 and SUZ12 were purchased from Abcam. 


\section{Cellular Physiology Cell Physiol Biochem 2018;46:1122-1133 \begin{tabular}{l|l} 
and Biochemistry Published onlıne: April18, 2018 & $\begin{array}{l}\text { (c) } 2018 \text { The Author(s). Published by S. Karger AG, Basel } \\
\text { www.karger.com/cpb }\end{array}$
\end{tabular} \\ Zeng et al.: LncDQ Promotes HCC Progression via EMT Pathway}

\section{Chromatin immunoprecipitation assays}

Chromatin immunoprecipitation (ChIP) assays were performed using the EZ-CHIP KIT (Millipore) according to the manufacturer's instructions. EZH2, H3 trimethyl Lys27, and SUZ12 antibodies were obtained from Abcam. Quantification of immunoprecipitated DNA was performed using qRT-PCR with the SYBR Premix ExTaq Kit (Takara). Data were calculated as a percentage relative to the input DNA.

Tumor growth and metastasis assays in nude mice

Male BALB/c athymic nude mice (5-week-old) were maintained in specific pathogen-free conditions and studied according to protocols approved by the Shenzhen Medical Experimental Animal Care Commission. Cells expressing sh-LncDQ or control vector were harvested and suspended in DMEM. Each mouse was injected subcutaneously into the right dorsal tissues or into the spleens with $5 \times 10^{6}$ cells in 200 $\mu \mathrm{L}$ medium. Then mice were sacrificed and examined for tumor growth and metastasis.

\section{Statistical analysis}

All statistical analyses were performed using SPSS 16.0 software (SPSS Inc, Chicago, IL). Statistical significance was analyzed using the Student's $t$-test, chi-squared test, or Fisher's exact test. The KaplanMeier method and log-rank test were used to estimate survival rates. A Cox proportional hazards model was used to calculate univariate and multivariate hazard ratios for the study variables. The receiver operating characteristics (ROC) curve was used to evaluate the effectiveness of LncDQ for prediction. $P$ values $<0.05$ were considered statistically significant.

\section{Results}

\section{LncDQ expression in HCC patients and cell lines}

The expression levels of LncDQ in 84 patients with HCC and paired non-tumor tissues were determined using qRT-PCR. As shown in Fig. 1A, LncDQ expression was significantly upregulated in HCC tissues samples compared to corresponding non-tumor tissues $(P$ $<0.001$ ). Furthermore, LncDQ expression was significantly upregulated in serum from patients with HCC $(n=50)$ compared with healthy controls $(n=30, P=0.0047)$ (Fig. 1B). Importantly, serum levels of LncDQ were positively associated with those of LncDQ in HCC tumor tissue $\left(r^{2}=0.731, P<0.01\right.$; Fig. 1C). Finally, LncDQ was also overexpressed in HCC cell lines compared to the normal human hepatic cell line L02 (Fig. 1D).

Fig. 1. Expression levels of LncDQ in HCC samples and cell lines. (A) Relative expression levels of LncDQ in HCC tissues compared with corresponding non-tumor tissues $(\mathrm{n}=$ 84). (B) Relative expression levels of LncDQ in HCC serum samples compared $(n=50)$ with healthy control serum $(n=30)$. (C) Correlation of LncDQ expression levels in HCC tissue and serum ( $\mathrm{n}=50)$. (D) Expression levels of LncDQ in HCC cell lines compared to normal cells. GAPDH was used as the endogenous control.

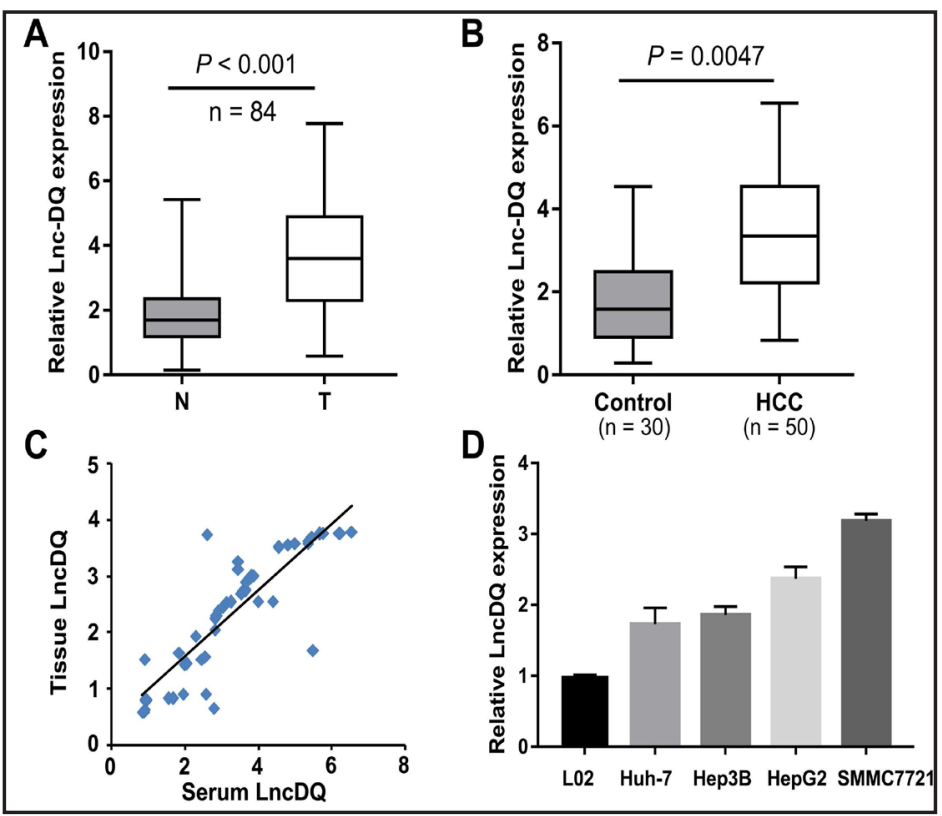


Correlation of $\operatorname{LncDQ}$ expression with clinicopathological characteristics

Next, we analyzed the relationship between LncDQ expression and the clinical features of HCC. According to LncDQ expression levels obtained by qRT-PCR, we divided the 84 HCC patients into a high LncDQ expression group $(n=58$, 1.5-fold higher than normal tissues) and lowLncDQ expression group $(n=26)$. Patients with high LncDQ expression exhibited a significant association with tumor stage $(P=0.003)$, lymph node metastasis $(P$ $=0.02)$, and tumor number $(P=0.014)$. However, there was no significant association between LncDQ expression and age, sex, AFP, differentiation, Hepatitis B/C infection, tumor size, venous invasion, or cirrhosis (Table 1).

\section{Upregulation of LncDQ was associated with poor prognosis in HCC patients}

Kaplan-Meier analysis and the log-rank test were used to analyze the relationship between LncDQ expression and patient survival. We found that the 5-year overall survival (OS) was significantly lower in patients with high LncDQ expression than in those with low LncDQ expression (29.3\% vs. $68.8 \%, P=0.001$; Fig. $2 A)$. Univariate analysis revealed that cirrhosis $(P=0.04)$, tumor stage $(P<0.001)$, lymph node metastasis $(P=0.001)$, venous invasion $(P=0.037)$, tumor number $(P=0.019)$, and LncDQ expression levels $(P=0.001)$ were significantly correlated with OS (Table 2 ). In addition, multivariate analyses indicated

Fig. 2. Upregulation of LncDQ was associated with poor prognosis and serum LncDQ levels as a diagnostic biomarker. (A) Patients with high LncDQ expression had poorer OS rates than did those in the low expression group $(n=84)$. (B) LncDQ expression was an independent prognostic indicator for OS. (C) ROC curve analysis was applied to determine the diagnostic value of LncDQ. (D) Serum LncDQ expression levels were downregulated in postoperative samples (n $=30$ ).
Table 1. Clinicopathological factors and LncDQ expression in HCC patients

\begin{tabular}{|c|c|c|c|c|c|}
\hline \multirow{2}{*}{ Characteristics } & & \multirow{2}{*}{ No. } & \multicolumn{2}{|c|}{ LncDQ } & \multirow{2}{*}{$P$ value } \\
\hline & & & low expression & high expression & \\
\hline \multirow[t]{2}{*}{ Age } & $<60$ & 42 & 15 & 27 & 0.345 \\
\hline & $\geq 60$ & 42 & 11 & 31 & \\
\hline \multirow[t]{2}{*}{ Gender } & Male & 47 & 16 & 31 & 0.49 \\
\hline & Female & 37 & 10 & 27 & \\
\hline \multirow[t]{2}{*}{ Tumor size } & $<5 \mathrm{~cm}$ & 53 & 16 & 37 & 0.843 \\
\hline & $\geq 5 \mathrm{~cm}$ & 31 & 10 & 21 & \\
\hline \multirow[t]{2}{*}{$\mathrm{AFP}$} & $<400$ & 29 & 12 & 17 & 0.133 \\
\hline & $\geq 400$ & 55 & 14 & 41 & \\
\hline \multirow[t]{3}{*}{ Differentiation } & Well & 37 & 13 & 24 & 0.408 \\
\hline & Moderate & 30 & 10 & 20 & \\
\hline & Poor & 17 & 3 & 14 & \\
\hline \multirow[t]{2}{*}{ Tumor stage } & $\mathrm{I} / \mathrm{II}$ & 35 & 17 & 18 & $0.003^{*}$ \\
\hline & III/IV & 49 & 9 & 40 & \\
\hline \multirow[t]{2}{*}{ Hepatitis B/C } & Negative & 14 & 4 & 10 & 0.833 \\
\hline & Positive & 70 & 22 & 48 & \\
\hline \multirow[t]{2}{*}{ Cirrhosis } & Negative & 40 & 14 & 26 & 0.444 \\
\hline & Positive & 44 & 12 & 32 & \\
\hline \multirow[t]{2}{*}{ Lymph node metastasis } & Negative & 64 & 24 & 40 & $0.02 *$ \\
\hline & Positive & 20 & 2 & 18 & \\
\hline \multirow[t]{2}{*}{ Venous invasion } & Negative & 44 & 16 & 28 & 0.261 \\
\hline & Positive & 40 & 10 & 30 & \\
\hline \multirow[t]{2}{*}{ Tumor number } & Single & 59 & 23 & 36 & $0.014^{*}$ \\
\hline & Multiple & 25 & 3 & 22 & \\
\hline
\end{tabular}

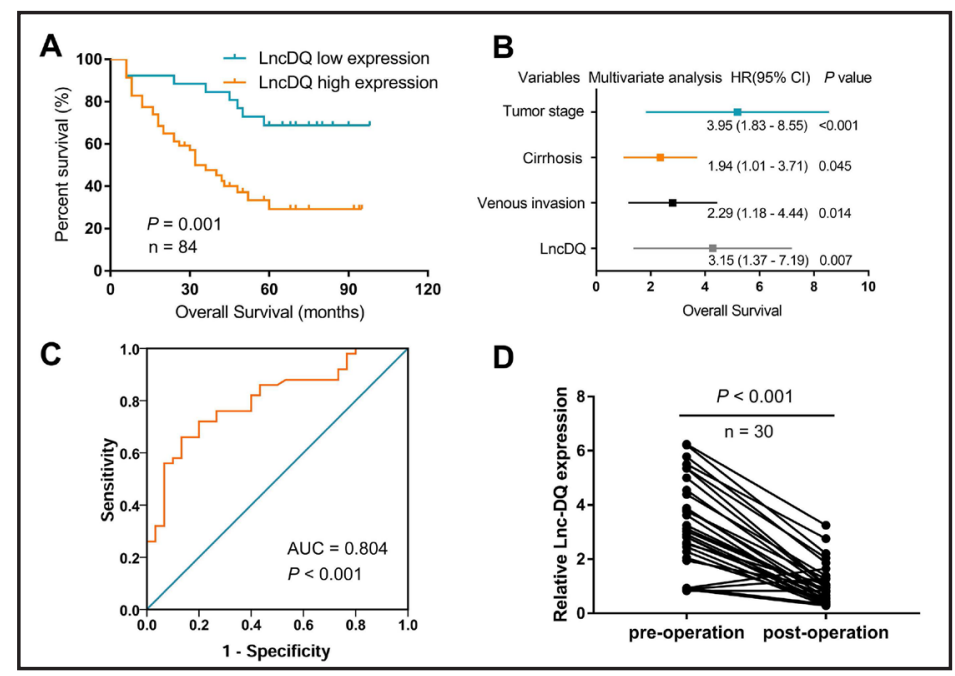


that LncDQ expression $(P=$ $0.007)$, cirrhosis $(P=0.045)$, tumor stage $(P<0.001)$, and venous invasion $(P=$ 0.014 ) were independent prognostic indicators for OS in HCC patients (Fig. 2B).

ROC curve of serum LncDQ level in the diagnosis of HCC

We further analyzed the ROC curve of serum LncDQ levels to assess its diagnostic value and found that serum LncDQ level could differentiate HCC patients from healthy controls (Fig. 2C), with an area under the curve (AUC) of 0.804 (95\% confidence interval [CI]: 0.707-0.9, $P<0.001$ ). LncDQ may be an effective predictor for HCC diagnosis, with an optimal cut-off value of 2.53 (sensitivity, 72\%; specificity, 80\%). Moreover, postoperative serum samples from 30 patients were collected 1 month after surgery. The expression levels of serum LncDQ in postoperative specimens significantly decreased compared with those in preoperative samples $(P<$ 0.001; Fig. 2D).

\section{Knockdown of $\operatorname{LncDQ}$ impaired HCC cell proliferation}

To confirm the functional role of LncDQ in HCC cells, we employed siRNA to specifically silence the expression of LncDQ in HepG2 and SMMC7721 cells (Fig. 3A-B). MTT assays were performed to evaluate the effects of LncDQ on HCC cell proliferation. LncDQ knockdown significantly suppressed cell proliferation compared with that in the control group (Fig. 3C-D). Consistent with this, anchorage-independent growth assays showed that clonogenic ability was significantly decreased after LncDQ knockdown (Fig. 3E).
Table 2. Univariate and multivariable Cox regression analyses for overall survival. $H R$ relative risk, 95\% CI 95\% confidence interval. * Statistically significant $P<0.05$, Cox proportional hazard regression model

\begin{tabular}{lcccccccc}
\hline \multirow{2}{*}{ Factor } & \multicolumn{4}{c}{ Univariate analysis } & \multicolumn{4}{c}{ Multivariate analysis } \\
& HR & $\mathbf{9 5 \%}$ CI & $\boldsymbol{P}$-value & HR & $\mathbf{9 5 \%}$ CI & \multicolumn{1}{c}{$\boldsymbol{P}$-value } \\
\hline Cirrhosis & 1.91 & 1.02 & 3.57 & $0.04^{*}$ & 1.94 & 1.01 & 3.71 & $0.045^{*}$ \\
Tumor stage & 3.68 & 1.80 & 7.53 & $<0.001^{*}$ & 3.95 & 1.83 & 8.55 & $<0.001^{*}$ \\
Lymph node metastasis & 3.28 & 1.71 & 6.32 & $0.001^{*}$ & & & & \\
Venous invasion & 1.91 & 1.03 & 3.55 & $0.037^{*}$ & 2.29 & 1.18 & 4.44 & $0.014^{*}$ \\
Tumor number & 2.16 & 1.16 & 4.04 & $0.019^{*}$ & & & & \\
LncDQ & 3.39 & 1.55 & 7.47 & $0.001^{*}$ & 3.15 & 1.37 & 7.19 & $0.007^{*}$ \\
\hline
\end{tabular}

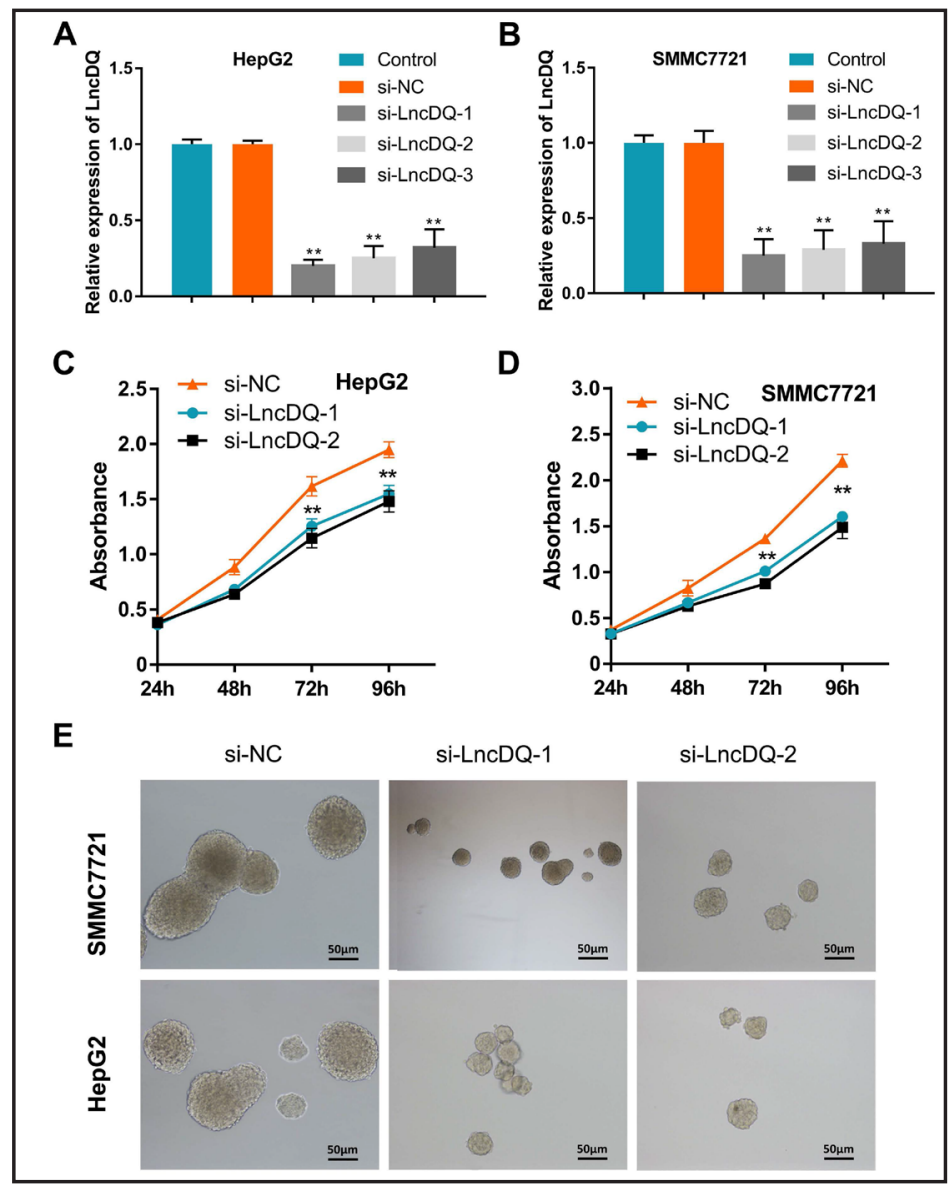

Fig. 3. LncDQ knockdown impaired cell proliferation. (A-B) Knockdown of LncDQ suppressed the proliferation of HepG2 and SMMC7721 cells. (C) Effects of LncDQ knockdown on the anchorage-independent growth of HepG2 and SMMC7721 cells. ${ }^{* *} \mathrm{P}<0.01$. 
Knockdown of $\operatorname{LncDQ}$ inhibited HCC cell migration and invasion

Next, we investigated the effects of LncDQ knockdown on cell migration and invasion by performing wound healing and transwell assays. Wound healing assays revealed that the downregulation of LncDQ led to a significant decrease in cell motility compared to that of the control cells (Fig. 4A-D). Similarly, the transwell invasion assay demonstrated that LncDQ depletion led to reduced cell invasion (Fig. 4E-H).

\section{Knockdown of LncDQ suppressed tumor progression in vivo \\ To investigate whether} LncDQ affected the progression of HCC in vivo, we evaluated the effects of LncDQ knockdown using a xenograft model. HCC cells stably transfected with shRNA targeting LncDQ or empty vector were injected subcutaneously into the right dorsal tissues of nude mice. Treatment with sh-LncDQ dramatically reduced tumor volume and weight compared with the control group (Fig. 5AC). Next, we determined the effects of LncDQ on intrahepatic metastasis in vivo by injecting LncDQ stable knockdown cells and control cells into the spleens of nude mice. Inhibition of LncDQ significantly reduced the incidence $(4 / 5$ in the LncDQ knockdown group and $1 / 5$ in the control group) and number of intrahepatic metastases (Fig. 5D-E).

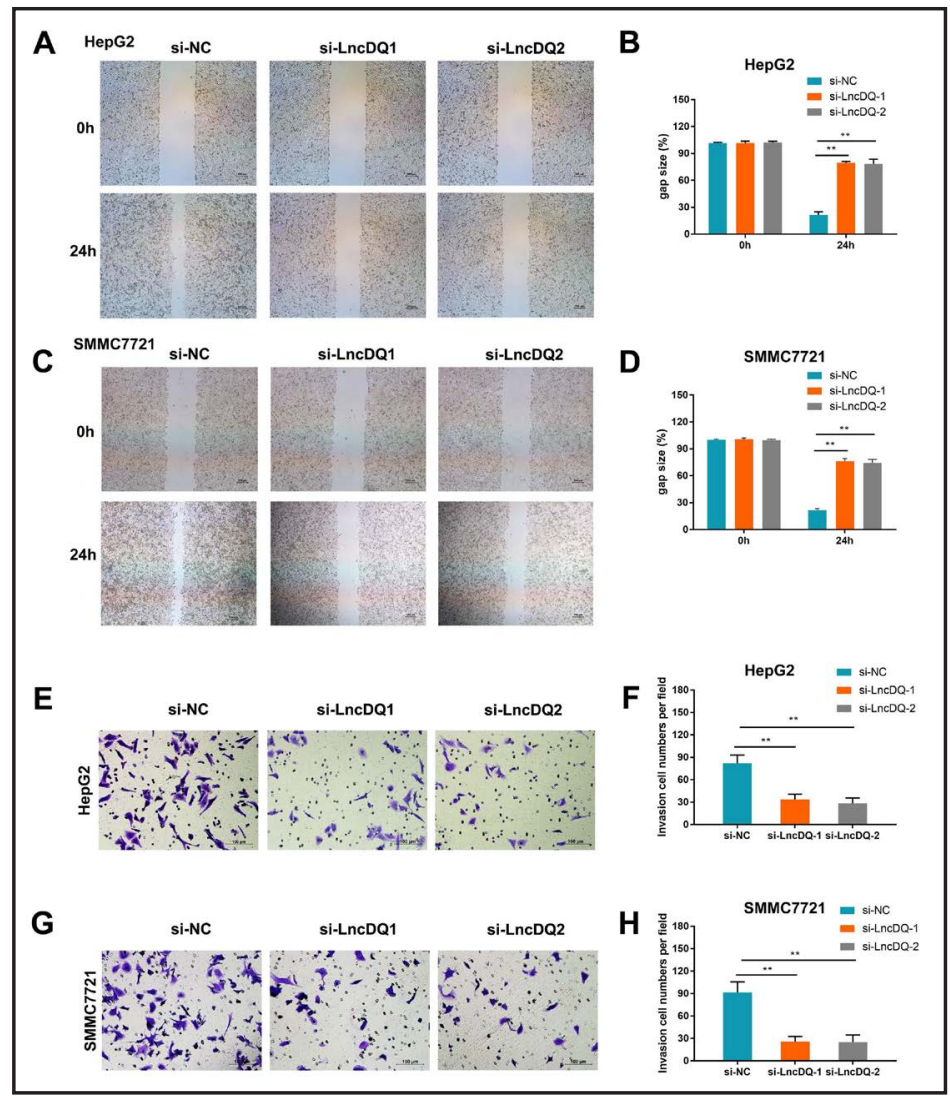

Fig. 4. LncDQ knockdown inhibited cell migration and invasion. (A-D) Effects of LncDQ knockdown on migration of HepG2 and SMMC7721 cells. (E-G) Knockdown of LncDQ suppressed the invasion of HepG2 and SMMC7721 cells. ${ }^{* *} \mathrm{P}<0.01$.

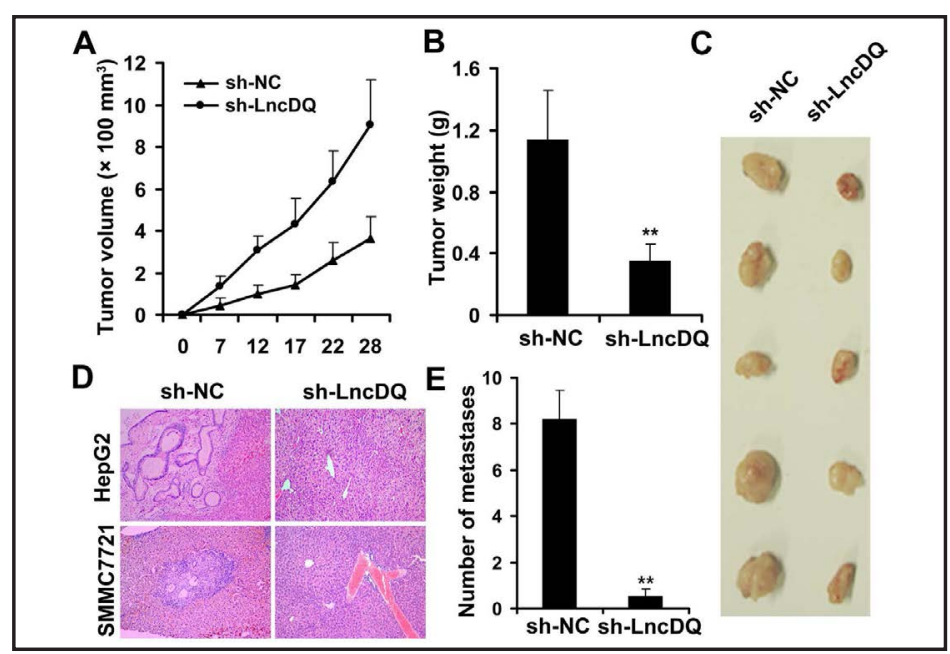

Fig. 5. LncDQ knockdown suppressed tumor growth and metastasis in vivo. (A) Growth curves of xenograft tumors after subcutaneous injection with shLncDQ and control cells. Tumor volumes were measured every 3 days after inoculation. (B) Tumor weights were measured after mice were sacrificed. Effects of LncDQ knockdown on tumor growth (C) and metastasis (D-E) in the xenograft models. ${ }^{* *} \mathrm{P}<0.01$. 
LncDQ regulated the EMT and epigenetically repressed E-cadherin expression by binding with EZH2

The EMT contributes to tumor progression in many cancers [18]. EZH2, a key subunit of polycomb repressive complex 2 (PRC2) that catalyzes the trimethylation of histone $\mathrm{H} 3$ at lysine 27 (H3K27me3), represses E-cadherin expression by interacting with LncRNAs in human cancer [19]. These studies prompted us to ask whether LncDQ promotes the EMT in HCC cells via PRC2-mediated histone modification. First, we evaluated expression of the EMT markers E-cadherin, N-cadherin, and vimentin by western blot analysis. The results showed that knockdown of LncDQ significantly decreased vimentin and $\mathrm{N}$-cadherin expression, and increased E-cadherin expression in HCC cells (Fig. 6A-B). Next, we determined the correlation between LncDQ and PRC2 by using an RIP assay in HCC cells. Interestingly, we found that endogenous LncDQ was dramatically enriched in the anti-EZH2 and anti-SUZ12 RIP fractions compared with the IgG fraction in both HepG2 and SMMC7721 cell lines (Fig. 6C-D). To further prove this hypothesis, we knocked down the expression of EZH2 in HCC cells using siRNA and observed that EZH2 knockdown led to a significant increase of E-cadherin expression (Fig. 7A-B). Finally, we performed a ChIP assay in LncDQ knockdown HCC cells, and found that knockdown of LncDQ reduced the binding of EZH2 and H3K27me3 levels at the E-cadherin promoter (Fig. 7C-D). Next, to investigate whether knockdown of LncDQ could affect expression of the PRC2 complex (EZH2 and SUZ12) and H3K27me3, we performed qRT-PCR and western blot assays. We found that si-LncDQ had no effect on the expression of EZH2 and SUZ12 (Fig. 8A-B), whereas the expression of H3K27me3 was reduced (Fig. 8CD). Taken together, these results suggested that LncDQ promoted the EMT and epigenetically regulated the expression of E-cadherin by interacting with EZH2.
B

D
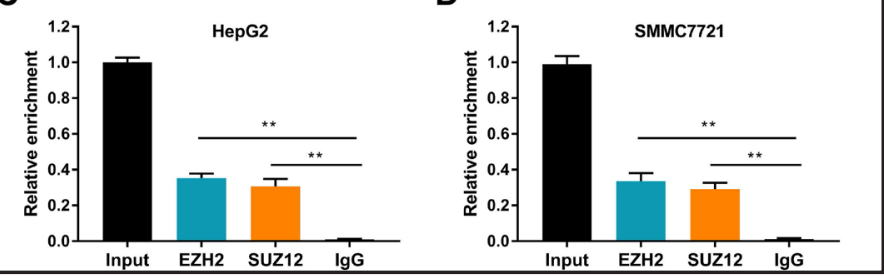

Fig. 6. LncDQ regulated the EMT and was associated with PRC2 in HCC cells. (A-B) Effects of LncDQ knockdown on vimentin, N-cadherin, and E-cadherin protein expression in HCC cells. (C-D) RIP experiments were performed and the co-precipitated RNA was subjected to qRTPCR for LncDQ. ${ }^{* *} \mathrm{P}<0.01$.

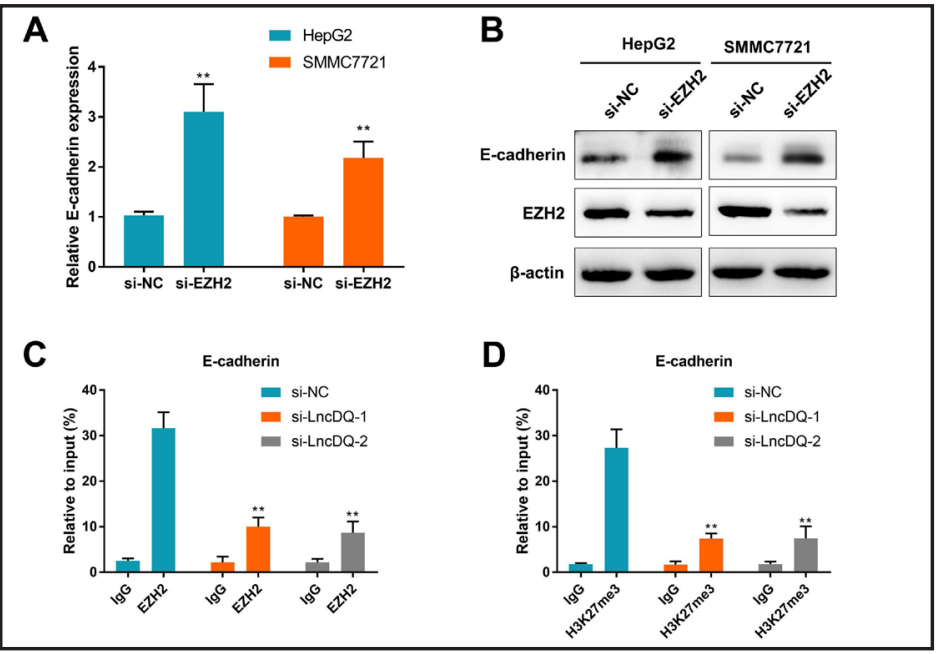

Fig. 7. LncDQ epigenetically repressed E-cadherin expression by interacting with EZH2. (A-B) Effects of LncDQ knockdown on E-cadherin expression were determined by qRT-PCR and western blot analysis. (CD) ChIP-qPCR was used to determine EZH2 and H3K27me3 occupancy of the promoter region of LncDQ in HepG2 cells. ${ }^{* *} \mathrm{P}<0.01$. 
Discussion

An increasing
number of reports have demonstrated that LncRNAs play an important role in tumor pathogenesis including HCC [20-23]. LncRNAs, especially circulating ones, are stable and accessible and are considered potential diagnostic or prognostic markers in some types of cancer [24-26]. Clinically, overexpression of LncDQ has been observed in active $\mathrm{CD}$ and correlates with disease severity [15]. A recent study demonstrated that high LncDQ expression contributes to the proliferation and metastasis of colorectal cancer in vitro and in vivo [16]. For HCC, a profile study found that LnCDQ was upregulated in HCC samples [14]. However, the role of LncDQ in HCC has not yet been explored. In this study, we found that LncDQ was upregulated in both HCC tissue samples and serum and was correlated with poor survival and adverse clinical pathological characteristics. Importantly, our results revealed that serum LncDQ levels could differentiate HCC patients from healthy controls with an AUC of 0.804. These results indicate that LncDQ can be used as a valuable diagnostic and prognostic marker for patients with HCC.

The EMT plays a crucial role during the progression of many types of cancer including HCC [27]. Recent studies have demonstrated that LncRNAs are also involved in regulation of the EMT pathway [28]. For instance, Wang et al. [28] revealed that taurine upregulated gene 1 (TUG1) is upregulated in colorectal cancer, and TUG1 knockdown reduced the migratory and invasive abilities of cancer cells by regulating the EMT progression. Cardiac hypertrophyrelated factor-mediated microRNA (miR)-489 suppression induced metastasis of colorectal cancer via the EMT pathway [29]. LncRNA-regulator of reprogramming (ROR) was shown to be upregulated in bladder cancer, and downregulated ROR reduced cell proliferation and metastasis by reversion of the EMT to mesenchymal-epithelial transition [30]. Unigene56159 can act as a competing endogenous RNA to decrease the expression of Slug by interacting with miR-140-5p [31]. In addition, upregulation of colon cancer-associated transcript 2 predicts poor prognosis in patients with prostate cancer and promotes metastasis by regulating the EMT [32]. In this study, we found that LncDQ promoted cell proliferation and motility via the EMT pathway, indicating that LncDQ acts as an oncogene in HCC. We show for the first time that LncDQ plays pivotal roles in the aggressive biology of HCC, which might help with the exploration of new therapeutic strategies for the treatment of HCC.

Studies have shown that many LncRNAs can physically interact with PRC2, and regulate target gene expression by recruiting PRC2 complexes to their promoters [33-35]. Interestingly, E-cadherin is also reportedly epigenetically repressed by the interaction of LncRNAs and EZH2 in several cancer types [36-38]. Our results showed that knockdown of LncDQ significantly increased E-cadherin expression in HCC cells, and endogenous LncDQ was enriched in anti-EZH2 RNA immunoprecipitation fractions. Furthermore, knockdown of EZH2 increased E-cadherin expression and reduced the binding of EZH2 and H3K27me3 
levels at the E-cadherin promoter. These results support the hypothesis that LncDQ promotes the EMT and epigenetically regulates the expression of E-cadherin by interacting with EZH2. Our results are consistent with previous studies, showing that LncRNAs can interact with PRC2 and epigenetically repress E-cadherin expression.

\section{Conclusion}

In conclusion, this study revealed that LncDQ is an independent prognostic factor for survival in HCC patients. Knockdown of LncDQ significantly suppressed cell proliferation, motility, and the EMT. LncDQ influenced the EMT by recruiting EZH2 to the E-cadherin promoter. Our study provides evidence supporting the critical role of LncDQ in HCC progression, and indicates that LncDQ expression may serve as a novel diagnostic and prognostic biomarker for HCC, thereby providing a potential therapeutic target for the control of disease progression.

\section{Abbreviations}

HCC (Hepatocellular carcinoma); LncRNAs (Long noncoding RNAs); EMT (Epithelialmesenchymal transition); LncDQ (LncDQ786243); PCR2 (Polycomb Repressive Complex 2); OS (Overall survival)

\section{Acknowledgements}

This work was financially supported by the Natural Science Foundation of Guangdong province (2015A030310099), and the National Natural Science Foundation of China (81401996), the Science and Technology Development Fund Project of Shenzhen (JCYJ2015040309144331).

BZ, CMT, and JKL conceived and designed the study. BZ, ZWL, and HLY performed the experiments and wrote the manuscript. JZ, DC, GTZ, XC, and JDZ analyzed and interpreted data. $\mathrm{ZFH}, \mathrm{MH}$, JZ, and CFC contributed the reagents and materials, and helped to draft the manuscript. All authors read and approved the final manuscript.

\section{Disclosure Statement}

No conflict of interests exists.

\section{References}

1 Yang JD, Roberts LR: Hepatocellular carcinoma: A global view. Nat Rev Gastroenterol Hepatol 2010;7:448458.

2 Llovet JM, Bruix J: Molecular targeted therapies in hepatocellular carcinoma. Hepatology 2008;48:13121327.

3 Farinati F, Marino D, De Giorgio M, Baldan A, Cantarini M, Cursaro C, Rapaccini G, Del Poggio P, Di Nolfo MA, Benvegnu L, Zoli M, Borzio F, Bernardi M, Trevisani F: Diagnostic and prognostic role of alpha-fetoprotein in hepatocellular carcinoma: Both or neither? Am J Gastroenterol 2006;101:524-532.

4 Batista PJ, Chang HY: Long noncoding rnas: Cellular address codes in development and disease. Cell 2013;152:1298-1307.

5 Huarte M: The emerging role of Incrnas in cancer. Nat Med 2015;21:1253-1261. 


\section{Cellular Physiology Cell Physiol Biochem 2018;46:1122-1133

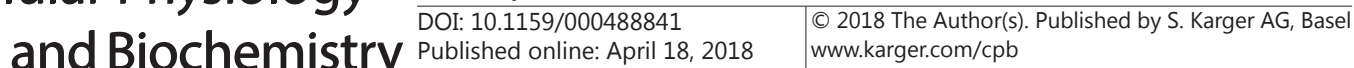

6 Adams BD, Parsons C, Walker L, Zhang WC, Slack FJ: Targeting noncoding rnas in disease. J Clin Invest 2017;127:761-771.

7 Li J, Tian H, Yang J, Gong Z: Long noncoding rnas regulate cell growth, proliferation, and apoptosis. DNA Cell Biol 2016;35:459-470.

8 Zeng B, Ye H, Chen J, Cheng D, Cai C, Chen G, Chen X, Xin H, Tang C, Zeng J: Lncrna tug1 sponges mir145 to promote cancer progression and regulate glutamine metabolism via sirt3/gdh axis. Oncotarget 2017;8:113650-113661.

-9 Schmitt AM, Chang HY: Long noncoding rnas in cancer pathways. Cancer Cell 2016;29:452-463.

10 Kang Y, Massague J: Epithelial-mesenchymal transitions: Twist in development and metastasis. Cell 2004;118:277-279.

11 Huang X, Zhi X, Gao Y, Ta N, Jiang H, Zheng J: Lncrnas in pancreatic cancer. Oncotarget 2016; 7:5737957390.

12 Wang J, Song YX, Wang ZN: Non-coding rnas in gastric cancer. Gene 2015;560:1-8.

13 Klingenberg M, Matsuda A, Diederichs S, Patel T: Non-coding rna in hepatocellular carcinoma: Mechanisms, biomarkers and therapeutic targets. J Hepatol 2017;67:603-618.

14 Yang F, Zhang L, Huo XS, Yuan JH, Xu D, Yuan SX, Zhu N, Zhou WP, Yang GS, Wang YZ, Shang JL, Gao CF, Zhang FR, Wang F, Sun SH: Long noncoding rna high expression in hepatocellular carcinoma facilitates tumor growth through enhancer of zeste homolog 2 in humans. Hepatology 2011;54:1679-1689.

15 Qiao YQ, Huang ML, Xu AT, Zhao D, Ran ZH, Shen J: Lncrna dq786243 affects treg related creb and foxp3 expression in crohn's disease. J Biomed Sci 2013;20:87.

-16 Sun L, Xue H, Jiang C, Zhou H, Gu L, Liu Y, Xu C, Xu Q: Lncrna dq786243 contributes to proliferation and metastasis of colorectal cancer both in vitro and in vivo. Biosci Rep 2016;36: e00328.

17 Liu G, Zhu J, Yu M, Cai C, Zhou Y, Fu Z, Gong Y, Yang B, Li Y, Zhou Q Lin Q, Ye H, Ye L, Zhao X, Li Z, Chen R, Han F, Tang C, Zeng B: Glutamate dehydrogenase is a novel prognostic marker and predicts metastases in colorectal cancer patients. J Transl Med 2015;13:144.

18 Liu S, Chen D, Shen W, Chen L, Yu A, Fu H, Sun K, Sun X: Ezh2 mediates the regulation of s100a4 on e-cadherin expression and the proliferation, migration of gastric cancer cells. Hepatogastroenterology 2015;62:737-741.

19 Luo M, Li Z, Wang W, Zeng Y, Liu Z, Qiu J: Long non-coding rna h19 increases bladder cancer metastasis by associating with ezh2 and inhibiting e-cadherin expression. Cancer Lett 2013;333:213-221.

20 Yuan JH, Yang F, Wang F, Ma JZ, Guo YJ, Tao QF, Liu F, Pan W, Wang TT, Zhou CC, Wang SB, Wang YZ, Yang Y, Yang N, Zhou WP, Yang GS, Sun SH: A long noncoding rna activated by tgf-beta promotes the invasionmetastasis cascade in hepatocellular carcinoma. Cancer Cell 2014;25:666-681.

21 Li H, Tang XM, Liu Y, Li W, Chen Q, Pan Y: Association of functional genetic variants of hotair with hepatocellular carcinoma (hcc) susceptibility in a chinese population. Cell Physiol Biochem 2017;44:447454.

22 Xiao J, Lv Y, Jin F, Liu Y, Ma Y, Xiong Y, Liu L, Zhang S, Sun Y, Tipoe GL, Hong A, Xing F, Wang X: Lncrna hanr promotes tumorigenesis and increase of chemoresistance in hepatocellular carcinoma. Cell Physiol Biochem 2017;43:1926-1938.

-23 Huang R, Wang X, Zhang W, Zhangyuan G, Jin K, Yu W, Xie Y, Xu X, Wang H, Sun B: Down-regulation of Incrna dgcr5 correlates with poor prognosis in hepatocellular carcinoma. Cell Physiol Biochem 2016;40:707-715.

24 Wang W, He X, Zheng Z, Ma X, Hu X, Wu D, Wang M: Serum hotair as a novel diagnostic biomarker for esophageal squamous cell carcinoma. Mol Cancer 2017;16:75.

25 Wan L, Kong J, Tang J, Wu Y, Xu E, Lai M, Zhang H: Hotairm1 as a potential biomarker for diagnosis of colorectal cancer functions the role in the tumour suppressor. J Cell Mol Med 2016;20:2036-2044.

26 Chen X, Lun L, Hou H, Tian R, Zhang H, Zhang Y: The value of Incrna hulc as a prognostic factor for survival of cancer outcome: A meta-analysis. Cell Physiol Biochem 2017;41:1424-1434.

27 Ibrahim AA, Schmithals C, Kowarz E, Koberle V, Kakoschky B, Pleli T, Kollmar O, Nitsch S, Waidmann O, Finkelmeier F, Zeuzem S, Korf HW, Schmid T, Weigert A, Kronenberger B, Marschalek R, Piiper A: Hypoxia causes downregulation of dicer in hepatocellular carcinoma, which is required for upregulation of hypoxiainducible factor 1alpha and epithelial-mesenchymal transition. Clin Cancer Res 2017;23:3896-3905.

28 Dhamija S, Diederichs S: From junk to master regulators of invasion: Lncrna functions in migration, emt and metastasis. Int J Cancer 2016;139:269-280. 


\section{Cellular Physiology Cell Physiol Biochem 2018;46:1122-1133

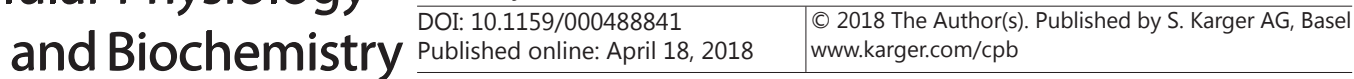 \\ Zeng et al.: LncDQ Promotes HCC Progression via EMT Pathway}

29 Tao Y, Han T, Zhang T, Ma C, Sun C: Lncrna chrf-induced mir-489 loss promotes metastasis of colorectal cancer via twist1/emt signaling pathway. Oncotarget 2017;8:36410-36422.

-30 Chen Y, Peng Y, Xu Z, Ge B, Xiang X, Zhang T, Gao L, Shi H, Wang C, Huang J: Lncror promotes bladder cancer cell proliferation, migration, and epithelial-mesenchymal transition. Cell Physiol Biochem 2017;41:23992410.

31 Lv J, Fan HX, Zhao XP, Lv P, Fan JY, Zhang Y, Liu M, Tang H: Long non-coding rna unigene56159 promotes epithelial-mesenchymal transition by acting as a cerna of mir-140-5p in hepatocellular carcinoma cells. Cancer Lett 2016;382:166-175.

-32 Zheng J, Zhao S, He X, Zheng Z, Bai W, Duan Y, Cheng S, Wang J, Liu X, Zhang G: The up-regulation of long non-coding rna ccat2 indicates a poor prognosis for prostate cancer and promotes metastasis by affecting epithelial-mesenchymal transition. Biochem Biophys Res Commun 2016;480:508-514.

33 Woo CJ, Maier VK, Davey R, Brennan J, Li G, Brothers J, 2nd, Schwartz B, Gordo S, Kasper A, Okamoto TR, Johansson HE, Mandefro B, Sareen D, Bialek P, Chau BN, Bhat B, Bullough D, Barsoum J: Gene activation of smn by selective disruption of lncrna-mediated recruitment of prc2 for the treatment of spinal muscular atrophy. Proc Natl Acad Sci U S A 2017;114:E1509-E1518.

-34 Portoso M, Ragazzini R, Brencic Z, Moiani A, Michaud A, Vassilev I, Wassef M, Servant N, Sargueil B, Margueron R: Prc2 is dispensable for hotair-mediated transcriptional repression. EMBO J 2017;36:981994.

-35 Wongtrakoongate P, Riddick G, Fucharoen S, Felsenfeld G: Association of the long non-coding rna steroid receptor rna activator (sra) with trxg and prc2 complexes. PLoS Genet 2015;11:e1005615.

-36 Chen S, Chen JZ, Zhang JQ Chen HX, Yan ML, Huang L, Tian YF, Chen YL, Wang YD: Hypoxia induces twistactivated epithelial-mesenchymal transition and proliferation of pancreatic cancer cells in vitro and in nude mice. Cancer Lett 2016;383:73-84.

-37 Zang C, Nie FQ, Wang Q, Sun M, Li W, He J, Zhang M, Lu KH: Long non-coding rna linc01133 represses klf2, p21 and e-cadherin transcription through binding with ezh2, lsd1 in non small cell lung cancer. Oncotarget 2016;7:11696-11707.

-38 Hirata H, Hinoda Y, Shahryari V, Deng G, Nakajima K, Tabatabai ZL, Ishii N, Dahiya R: Long noncoding rna malat1 promotes aggressive renal cell carcinoma through ezh2 and interacts with mir-205. Cancer Res 2015;75:1322-1331. 